\title{
LA VIOLENCE DANS LES FAMILLES RECOMPOSÉES
}

\author{
A VIOLÊNCIA NAS FAMÍLIAS RECOMPOSTAS
}

\begin{abstract}
Nicole Stryckman*
RÉSUMÉ: Ce que je vais avancer est le constat de ma pratique psychanalytique d'adultes et du travail effectué avec des psychanalystes d'enfants. Les processus évoqués ainsi que les affects se situent au niveau de l'inconscient. Ces processus, qui relèvent du désir, des pulsions et de la jouissance, vont se faire entendre dans les ratages de ce discours, dans les rêves et dans la formation de symptômes dans le corps et/ou dans la pensée. Les effets des violences que je vais souligner sont donc, au départ, inconscientes et ne sont qu'une facette de ce prisme très complexe qu'est notre structure corporelle et psychique. Ce que j'avance ici n'est qu'une des dimensions de la constitution de notre subjectivité. De plus, dans cette dimension inconsciente, j'ai choisi la violence. Je vais d'abord spécifier ce qu'est la violence auquel je me réfère et la différencier de l'agressivité et de la haine. Ensuite, je vais poser la question: Quelles sont les formes de violences exercées dans toutes les familles et reprises parfois redoublées dans les familles "recomposées" en insistant plus particulièrement sur la violence de l'oubli et celle de l'originaire. Pour terminer, je tenterai d'ébaucher les remèdes possibles à ces violences et le bon usage possible de celles-ci par le beau- parent.
\end{abstract}

MOTS CLÉS: Psychanalyse. Violence. Familles recomposées.

RESUMO: O que vou avançar é constatação de minha prática psicanalítica com adultos e do trabalho efetuado com psicanalistas de crianças. O processo evocado, assim como os afetos, situam-se na dimensão do inconsciente. Estes processos que são parte desejo, das pulsões e do gozo, vão se fazer compreender nas falhas deste discurso, nos sonhos e na formação de sintomas no corpo e/ou no pensamento. Os efeitos das violências que vou assinalar são então, inicialmente, inconscientes, e são apenas uma faceta deste prisma muito complexo que é nossa estrutura corporal e psíquica. O que avanço aqui é apenas uma das dimensões da constituição de nossa subjetividade. Além disso, nesta dimensão inconsciente escolhi a violência. Assim, vou primeiro especificar o que é violência à qual me refiro e diferencia-la da agressividade e do ódio. Em seguida, vou colocar a questão: quais são as formas de violência exercidas em todas as famílias e ás vezes reduplicadas nas famílias "recompostas" insistindo mais particularmente sobre a "violência do esquecimento" e uma outra, a "do originário". Para concluir, tentarei delinear alguns remédios para essas violências e o bom uso possível por padrastos e madrastas.

PALAVRAS-CHAVE: Psicanálise. Violência. Famílias recompostas.

* Psicanalista da Association Espace Analytique de Paris.

1 Ecrit à partir d'une communication à la IVème Journée scientifique de l'Institut de formation du CEFA sur "Les familles recomposées", à Bruxelles, le 19 octobre 1994. 
"Tout choix ultérieur d'amitié et d'amour se fait sur fond de traces mnésiques laissées par ces premiers modèles."

S. FREUD. ${ }^{2}$

Pour la bonne compréhension de mon propos, je voudrais, à titre de préliminaires, vous demander de bien garder à l'esprit deux choses:

I. Ce que je vais avancer est le constat de ma pratique psychanalytique d'adultes (qui tous ont été des enfants) et du travail effectué avec des psychanalystes d'enfants. Les processus évoqués ainsi que les affects se situent au niveau de l'inconscient c.à.d. dans un lieu de notre corps et de notre psychisme qui nous est à nous même inconnu et qui ne se dévoile, ni ne s'énonce pas dans le discours commun. Ces processus, qui relèvent du désir, des pulsions et de la jouissance, vont se faire entendre dans les ratages de ce discours, dans les rêves et dans la formation de symptômes. Symptôme dans le corps et/ou dans la pensée. Les effets des violences que je vais souligner sont donc, au départ, inconscientes et ne sont qu'une facette de ce prisme très complexe qu'est notre structure corporelle et psychique.

2. Nous sommes tous des sujets déterminés par notre inconscient, mais nous ne sommes pas que cela et donc, ce que j'avance ici n'est qu'une des dimensions de la constitution de notre subjectivité. De plus, dans cette dimension inconsciente, je n'ai choisi qu'un point particulier: la violence. Il y en a bien d'autres, par exemple, l'identité sexuelle, les diverses identifications, les désirs parentaux, les conditions socioéconomiques, etc.

Très brièvement, je vais d'abord spécifier ce qu'est la violence auquel je me réfère et la différencier de l'agressivité et de la haine.

Ensuite, je vais poser la question suivante:

Quelles sont les formes de violences exercées dans toutes les familles et reprises parfois redoublées dans les familles "recomposées" 3 , en insistant plus particulièrement sur la violence de l'oubli et celle de l'originaire. Pour terminer, je tenterai d'ébaucher les remèdes possibles à ces violences et le bon usage possible de celles-ci par le beau- parent.

\section{VIOLENCE}

La violence est difficile à définir alors qu'il est aisé de l' identifier. Elle comporte plus d'une face: notamment la violence physique qui vise la destruction des corps et des

2 Freud S., Sur la psychologie du lycéen. In Résultats, idées, problèmes. Paris, Puf, tome I, p. 230.

3 Terme qui ne me paraît pas fort adéquat dans la mesure où il fait penser à un retour de l'ancienne famille. Je l'utilise néanmoins puisqu'aucun autre plus indiqué n'a pu s'imposer à ce jour. 
objets, la violence économique qui vise l'exploitation de la force de travail et la violence psychique qui vise la destruction de l'intégrité psychique c.à.d. de l'intégrité désirante et sexuée du sujet.

Nous n'envisagerons aujourd'hui que cette violence psychique. Remarquons tout d'abord qu'elle s'exerce en deux lieux différents.

a. Le lieu de l'extra-psychique, autrement dit le lieu du rapport du sujet avec l'Autre ${ }^{4}$ et avec ses semblables (parents, enfants, beaux-parents, beaux-enfants, etc.).

b. Le lieu de l'intra-psychique. Il s'agit là de la violence qui s'exerce entre les différentes instances psychiques telles que le Moi, le Ca et le Surmoi et les lieux de leur exercice: le conscient, le préconscient et l'inconscient, pour le dire en termes freudiens ou, en termes lacaniens: le réel, le symbolique et l'imaginaire et le lieu de l'Autre.

La clinique psychanalytique nous apprend que l'inconscient fait violence à l'être humain, car il détermine à l'insu du sujet, ses affects, ses représentations de lui- même, de son corps et du monde, ainsi que sa vie fantasmatique, son mode de pensée, ses désirs et ses jouissances. Il suffit de nous regarder vivre dans nos amours, nos haines, nos histoires familiales etc... Ces histoires nous démontrent chaque fois que l'origine de la vie et du désir, dans l'inconscient, restent marquées par l'inceste et son interdit. Reconnaître cette marque incestueuse de l'amour et de la haine que véhiculent ces origines permet de mieux appréhender leurs effets dans le champ des représentations, des désirs et des actions des parents et des enfants lors des ruptures et des divorces.

Remarquons que tout être humain se fait d'abord violence à lui-même. Notons par ailleurs, que contrairement à l'agressivité et à la haine, la violence n'a pas nécessairement un objet précis. Disons qu'elle n'est pas sans objet. Ce en quoi elle est proche de l'angoisse.

Mais si elle n'a pas un objet précis, elle a par contre une visée très précise: elle vise toujours à détruire ce qui met en danger la vie du sujet. Elle est une réponse dans la réalité de la vie, à une situation de danger de mort réelle ou psychique, danger venu de l'extérieur ou perçu comme tel.

Comme nous le verrons, la violence est une dimension inhérente, constitutive de la vie puisque celle-ci s'origine dans la violence, celles du rapport sexuel, de l'accouchement, de l'entrée dans le langage etc... La violence se situe dans le réel de l'existence et s'exerce par des mises en acte.

La clinique psychanalytique ajoute qu'elle est souvent mise en acte de la haine.

4 Concept utilisé par J. Lacan pour désigner le lieu où la parole se constitue et inscrit le sujet dans son être de désir et dans sa subjectivité inconsciente. 


\section{AGRESSIVITÉ}

L'agressivité est un processus psychique impliquée dans la constitution de l'image de soi. Elle s'origine d'abord dans l'imaginaire. Pour Lacan, l'agressivité est “ constitutive de la première individuation subjective". ${ }^{5}$

Les effets, dans les familles recomposées de cette agressivité pour l'autre du miroir sont évidents. Il nous suffit de regarder les rapports de jalousies et de rivalités entre frères et soeurs, entre enfants et parents ou entre beaux-parents et beaux-enfants.

L'agressivité a un objet précis, c'est l'autre, mon semblable. Sa visée est l'emprise sur cet autre ou l'appropriation de ses objets. Bien sûr, elle comporte aussi une tendance à la destruction de l'autre: sa mort, sa disparition, est le meilleur moyen pour s'assurer la propriété de ses objets.

La clinique psychanalytique nous le démontre: l'agressivité peut aussi être source de plaisir, voire de jouissance. Comme l'indiquait déjà Freud, elle peut être un plaisir auquel l'homme renonce difficilement dès qu'il y a goûté.

J'ajouterai enfin que l'agressivité contient la haine et ce, dans le double sens du terme contenir. D'une part, la haine s'y trouve incluse. D'autre part la haine y est endiguée, limitée. L'agressivité permet son expression de manière acceptable pour soi et pour l'autre.

\section{HAINE}

Il n'existe pas de distinction claire et constante dans la théorie psychanalytique entre haine et agressivité. Néanmoins, si nous nous référons à la structure du sujet et au nouage qu'elle implique du réel, de l'imaginaire et du symbolique nous pouvons dire avec Lacan, que la haine se situe à la conjonction du réel et de l'imaginaire, tandis que l'amour se situe au carrefour du symbolique et de l'imaginaire ${ }^{6}$. Je vous propose donc de différencier la haine de l'agressivité comme elle se différencie de l'amour.

Elle n'est pas l'envers de l'amour, c'est une illusion que de le penser. La haine n'est pas un amour négatif. Par ailleurs, la haine se distingue de l'agressivité. La haine implique un "vouloir" détruire. La haine a comme visée, comme but, de supprimer non seulement l'image de l'autre, mais son être même. Comme je l'ai indiqué dans une intervention précédente ${ }^{7}$, "la haine ne vise pas la destruction de l'image spéculaire, mais bien la destruction du défaut constitutif de cette image". J'avancerai avec G. Le Gaufey

5 Lacan J., L'agressivité en psychanalyse, in Ecrits, Paris, Seuil, 1966, p.ll7.

6 Lacan J. Séminaire I. Leçon du 30 juin 1954. Seuil, 1975, p. 298.

7 Stryckman N., Dis moi, miroir, suis-je la plus belle du Royaume? Communication à la Journée de fin d'année de l'Association freudienne de Belgique, à Bruxelles en juin 1993. 
que "la haine cherche à anéantir l'appartenance symbolique du sujet au-delà du spéculaire". Autrement dit, c'est non seulement l'être de désir qui est visé mais au-delà, les géniteurs d'où le désir s'engendre. Dans les familles recomposées, cette haine pourrait avoir, inconsciemment comme mire le désir qui a donné naissance à cet enfant. Détruire le lieu de l'origine dans sa dimension imaginaire en visant par exemple le partenaire exclu; dans sa dimension symbolique en visant par exemple le nom de l'enfant, dans sa dimension réelle en visant par exemple, le lieu de vie de l'enfant.

La haine est donc une intrication de l'imaginaire et du réel.

D'un point de vue psychanalytique, la haine provient de la lutte du moi pour la conservation de la vie et son affirmation. Une des découvertes de Freud fut de constater que la haine est plus ancienne que l'amour et qu'elle est une réponse à la domination exercée sur l'enfant par la toute puissance qu'il supposait à sa mère. Mais la haine peut aussi s'originer dans la perte de cette toute puissance maternelle et, corrélativement, à la perte de sa propre toute puissance, celle de "his majesty the baby".

C'est sans doute ceci que revivent les enfants lors de la séparation de leur parents et qui les amène à rendre consciemment ou inconsciemment l'impuissance de leur mère responsable de cette séparation même si c'est le père qui la souhaite et qui en prend l'initiative. Aux yeux de l'enfant, la mère continue d'être investie fantasmatiquement et pendant un long temps, de cette toute-puissance qu'elle a effectivement exercée sur lui, sur sa vie même, pendant le temps de la grossesse et de l'accouchement. Et donc, l'enfant attend de sa mère, qu'elle ait tout pouvoir sur le père pour le garder à la maison. Là où lui même a échoué, il attend, il exige de sa mère, de cet Autre-Réel qu'elle réussisse. Cette mise en échec de la mère et de lui-même, ranime en lui le double deuil qu'il a ou qu'il est en train d'accomplir:

1. Celui d'avoir une mère toute-puissante qui peut tout pour lui, deuil nécessaire pour qu'il puisse se séparer d'elle. Ce travail de deuil implique pour l'enfant le meurtre de ce fantasme. Ce meurtre métaphorique, bien sûr, peut produire chez l'enfant un sentiment de culpabilité difficilement contournable. Pour apaiser, voire pour supprimer cette culpabilité, l'enfant "choisira" dans certains cas d'établir un rapport masochiste à l'égard de sa mère ou de lui vouer une haine sans nom.

2. Second deuil: celui de la première représentation de lui même. Celle-ci peut-être l'enfant merveilleux ou terrifiant qui témoigne des rêves et désirs des parents. Comme le rappelle S. Leclaire, “...il n'est de vie qu'au prix du meurtre de l'image première étrange, dans laquelle s'inscrit le narcissisme de chacun". Car il ne suffit pas de tuer les images parentales mais "encore faut-il tuer la représentation tyrannique de l'enfant-roi". ${ }^{9}$ La toute-puissance supposée à la mère comme la première image

\footnotetext{
8 Au sens où Freud a parlé du choix de la névrose.

9 Leclaire S., On tue un enfant. Paris, Seuil, 1975, p. 11.
} 
narcissique que l'enfant a de lui-même vont être reconvoquées lors de ces séparations et lors de cette recomposition familiale.

Par ailleurs, le départ du père resignifie à l'enfant la perte de sa toute-puissance comme la perte de celle de sa mère. Cette double perte peut engendrer la haine. Mais l'enfant n'ose l'adresser à son père ni à sa mère, car il a besoin de leur amour et de leur reconnaissance. Une des issues consiste à la retourner contre lui-même.

La haine contre soi comme réaction à une grande détresse peut s'exprimer par ces mots combien de fois entendu dans les discours d'adultes: "Je ne m'aime pas, je hais ce que je fais, j'ai honte de moi,..."

L'enfant attendra adoucissement de cette haine par l'amour maternel et par l'environnement familial. Vous pouvez facilement supposer que cette haine sera présente lorsque la belle-mère viendra prendre la place de la mère et ainsi signifier à nouveau à l'enfant l'impuissance de sa mère à se faire désirer par le père ainsi que la perte de sa place d'enfant-roi. Le belle-mère se présente comme un nouvel idéal pour l'enfant, puisqu'elle vient pallier au manque du premier idéal qu'était sa mère.

Nous avons ici, l'exemple d'un processus qui crée ou recrée un sentiment de haine et aussi l'explication du fait couramment constater que la séparation constitue pour l'enfant une véritable crise subjective c'est à dire, la conjonction de deux faits. "Il faut, d'une part, qu'une rupture se produise dans la représentation signifiante par laquelle le sujet est inscrit dans le grand Autre en tant que lieu de code. Il faut d'autre part, qu'à partir de cette rupture, le discours qui s'adresse au sujet modifie ses choix identificatoires qu'il mette en question son idéal du moi et enfin que son objet d'amour se refuse à lui comme objet de jouissance". ${ }^{10}$

Dans la mesure où la belle-mère, du fait de sa structure psychique, et du rapport affectif qu'elle a noué avec le père de l'enfant et sa mère, va effectivement authentifier dans le réalité qu'elle est toute-puissante, qu'elle a réussi, ceci va inviter l'enfant, dans son fantasme et son processus d'idéalisation, à substituer sa belle-mère à sa mère et inévitablement surgiront des sentiments d'infidélité à l'égard de l'amour pour la mère, une interrogation sur son identité, une émergence de sentiments de culpabilité et dans certain cas une mise en danger de son intégrité psychique. Comme je vous l'ai déjà avancé, c'est lorsque ce danger surgit que la violence peut éclater. "Pris au piège des idéaux, tout ce qui serait alors signe de faille ou de différence viendrait briser ces illusions et pourrait être vécu comme une violence. Car si l'amour tente d'adoucir voire parfois de détruire la différence, la haine témoigne de l'insupportable de cette différence."11

\footnotetext{
${ }^{10}$ Stryckman N. Parents en crise, adolescent en "passe". In La psychanalyse de l'enfant. Paris, Ed. Association freudienne, 1989, Tome 2, p. 56.

11 Descamps M. La fonction parentale au risque des parentés plurielles. In Steichen R. et De Neuter P. (édit), Les familles recomposées et leurs enfants. Louvain-la-Neuve, Académia, 1995, pp. 145-146.
} 


\section{QU'EST-CE QUI DANS LES RAPPORTS ENFANTS-PARENTS, BEAUX- ENFANTS-BEAUX-PARENTS FAIT SURGIR LA VIOLENCE?}

"Quelque soit la culture, écrit B.Cyrulnik, la famille demeure le lieu de la violence"12. Ce qui fait surgir la violence dans la famille c'est la non correspondance entre les désirs individuels et la volonté générale. La fonction du couple et de la famille consiste à rendre compatible ces deux éléments. Ceci tend à se faire au sein des relations d'amour et de haine qui constituent le tissu nécessairement conflictuel du groupe familial.

Or, la famille "recomposée", du fait même de cette recomposition, réinterroge et remet en jeu ces mécanismes, ces processus d'amour et de haine. La remise en jeu de ces dialectiques va redoubler d'intensité du fait que cette reconstruction du couple et de la famille n'emporte l'adhésion ni des enfants, ni du parent exclu. Par conséquent, cette nouvelle cellule familiale ne peut pas jouer ce rôle de rendre compatible ces deux fonctions. Au contraire, elle en souligne plutôt la non correspondance, voire l'opposition entre les désirs individuels et la volonté générale. Ceci rend inévitable l'émergence de la violence, sinon dans les faits, du moins dans les coeurs. Cette violence est surdéterminée par toutes les précédentes et revisitées par cette recomposition. C'est ce qui nous fait dire qu'elle est redoublée.

Anne-Marie Decuypere a proposé une vignette clinique illustrant bien ce redoublement. Tatiana, vient consultée à 21 ans de sa propre initiative. Elle souffre de violentes crises d'angoisses. Ses parents se sont séparée 18 mois après sa naissance. Bien que le traumatisme de la naissance se soit trouvé noué au traumatisme de la séparation, ce n'est qu'au moment du remariage de sa mère que ses crises se déclenchent de par ses incidences rétroactives sur le trauma initial. "Cela se passe comme si, actuellement, du fait d'être confrontée au 'départ' de sa mère, dont le désir se porte vers un autre homme, la jeune fille vivait dans l'après coup, les effets pénibles d'un trauma ancien, qu'elle n'avait pas vécu comme tel. "13 Par ailleurs, il y a des violences spécifiques à la recomposition familiale qui vont s'inscrire et s'ajouter aux premières, voilà un second redoublement des violences.

\section{QUELLES SONT LES VIOLENCES AUXQUELLES LES PARTENAIRES DES FAMILLES RECOMPOSÉES VONT SE HEURTER INÉVITABLEMENT?}

Envisageons d'abord celle que nous avons déjà évoquées: la violence de l'inconscient et ici, plus particulièrement, celle des origines avec les effets sur la

\footnotetext{
${ }^{12}$ Cyrulnik B., Les nourritures affectives, 1993, O. Jacob, p.131.

13 Decuypere A., Les nouvelles compositions familiales et la question du temps". In Steichen R. et De Neuter P., op. cit., p. 194.
} 
constitution de l'identité de l'enfant. Les psychanalystes appellent parfois cette violence le "traumatisme de la naissance". Elle sera suivie par celle qu'implique tout apprentissage. Tout ceci va se réactualiser lors de la recomposition.

Il y a aussi la violence de l'oubli, notamment celui de la séparation, de la rupture et des événements qui ont précédés et suivis cette décision.

On ne peut enfin ignorer la violence des deuils à faire vu les séparations inévitables dont l'enfant attribuera parfois la responsabilité à tort ou à raison au beau-parent introduit comme membre du nouveau groupe familial.

\section{Y A-T-IL DES VIOLENCES SPÉCIFIQUES A LA RECOMPOSITION FAMILIALE?}

Oui, assurément et j'en distinguerai trois:

- La violence de la rupture de l'unité familiale.

- La violence du rejet, voire des sentiments d'agressivité, d'un des deux parents, rejet ou sentiments réels ou perçus comme tel.

- La violence du lieu de l'originaire de l'enfant. D'une part par le clivage de ce lieu - deux couples, deux familles -. D'autre part, par les effets que ce clivage ainsi que la désunion de la première famille peut opérer sur l'identité de l'enfant et ce, de diverses manières.

Arrêtons-nous à deux modalités de cette violence.

\section{LA VIOLENCE DE L'OUBLI.}

Cette violence ne s'exerce pas dans l'oubli normal et légitime dans lequel nous laissons tomber beaucoup d'événements de la vie. Car nous ne pouvons pas vivre en permanence sous l'effet de souvenirs douloureux et déchirants. L'oubli apaise les blessures. Il est nécessaire.

Par violence de l'oubli j'entends celle qui vise à chasser, à dénier, les événements douloureux vécus ensemble. Cette violence permet de ne pas assumer la part que l'on a eu dans ces événements ainsi que celle que l'on exige que l'autre assume: l'ex-conjoint ou les enfants. Au partenaire qui ne souhaitait pas cette séparation, à l'enfant qui devra davantage se prendre en charge, passer d'un lieu de vie à un autre, perdre la sécurité affective que procure l'union du couple parental et souvent ne plus avoir de contact avec l'un de ses parents. Je me souviens de cette parole adressée à une petite fille de 7 ans: “Ton père, tu oublies. Il m'a quittée et donc par conséquence, il t'a laissée tombée. Ton père, c'est terminé". Parfois violence est faite aussi au nouveau compagnon à qui il est demandé de réparer les pots cassés d'un ménage qui n'était pas le sien en oubliant sa propre histoire. A d'autres, on demande d'être celui qui va permettre que se recrée une famille idéale, c'est à dire impossible. 
Dans cette conjoncture, l'exigence de l'oubli vise parfois à ne pas reconnaître la violence qui fut exercée par le passé afin de pouvoir la répéter secrètement et de manière souterraine." Ah! cette exquise violence quotidienne", s'exclamait un jour un analysant.

On peut, me semble-t-il, comprendre le rôle capital que le beau-parent peut jouer par rapport à tout ceci.

Il arrive que le beau-parent demande à l'enfant de tourner la page sur sa vie passée et suivant son âge, sa structure psychique, les circonstances familiales, le déclin ou non de la problématique oedipienne, l'enfant le fera ou ne le fera pas. Mais ainsi le beau-parent déclenche, sans le savoir peut-être, un processus de tentative d'oubli voire de refoulement de ce passé qui va inévitablement faire retour dans des affects et/ou des actes violents. Car l'enfant ressent que ses origines sont attaquées, voire refusées, que son identité est mise en danger et qu'on lui demande de devenir un autre enfant.

Il arrive aussi que le beau-parent reconnaisse ce passé et l'inclut dans son rapport à l'enfant, tout en respectant sa place d'étranger dans ce passé. Il aura le plaisir de constater que cela va apaiser l'enfant et que celui-ci, progressivement, y fera de moins en moins référence. Car reconnaître le passé comme passé permet de ne plus le vivre comme présent.

\section{VIOLENCE DE L'ORIGINAIRE}

J'insiste sur cet originaire, car le beau-parent, vu la place qu'il occupe pour l'enfant, a pris celle du parent absent et donc a fait violence à l'unité de la cellule familiale, à l'unité du couple, à ce lieu sécurisant pour l'enfant, à ce lieu à partir duquel il construit son identité, forge ses idéaux et ses identifications et arrime ses désirs. Bref, il a fait violence à cette dimension symbolique de son être en voie de construction, à qui il demande une reconstruction.

Ce qui a présidé à la conception et à la venue d'un enfant, c'est ce que nous psychanalystes nous appelons la scène primitive, où s'est mise en acte, dans un rapport au sexe, une histoire d'amour, de désirs et de jouissances, désirs d'enfants et désir de filiation. De cette scène primitive, soulignons deux choses, car elles vont poursuivre le sujet toute sa vie et seront particulièrement présentes inconsciemment bien sûr, dans les rapports avec le beau-parent puisqu'il prend place au lieu qui a présidé à sa conception et, d'une certaine façon, le détruit.

Or cette scène primitive est toujours imaginée par l'enfant comme doublement violente:

- Il l'imagine comme violence faite à la mère. Ce qui va l'exciter et éveiller sa sexualité, nouvelle violence pour lui, inscrite dans son corps biologique.

- Cette scène primitive est encore violence pour l'enfant lui même, au sens ou de ce lieu de sa conception, il est absent. Comme le souligne admirablement P. Quignard: "Nous transportons avec nous le trouble de notre conception. 
Il n'est point d'image qui nous choque qu'elle ne nous rappelle les gestes qui nous firent.

Or, cette "chose regardée en même temps", nous ne pouvons en aucun cas la voir.

Nous sommes venus d'une scène où nous n'étions pas.

L'homme est celui à qui une image manque.

L'homme est un regard désirant qui cherche une image derrière tout ce qu'il voit." ${ }^{14}$

Une question s'impose ici: comment renoncer à quelque chose auquel nous n'avons pas accès?

L'arrivée du beau-parent et de surcroît la naissance d'un enfant du nouveau couple vient raviver ces blessures anciennes et ce renoncement impossible. Cette arrivée et cette naissance vont interroger l'identité que l'enfant à reçu de fait (tel le nom, le prénom), son identité sexuelle et les interdits fondateurs. Mais aussi, l'identité que l'enfant s'est forgé dans le rapport avec ses parents et sa fratrie. Identité construite dans un rapport de reconnaissance narcissique et narcissisante de l'enfant par le parent et du parent par l'enfant.

Or, le lien avec le beau-parent va souvent s'instaurer dans un rapport de séduction qui cette fois sera sexué et dans certains cas érotisée comme le rappelait J.P. Winter dans sa conférence sur "L'érotique dans la famille recomposée". ${ }^{15}$ Cependant ce nouveau rapport ne pourra se réaliser que sur le modèle du premier et donc en rappeler l'effectuation. Cela aura pour conséquence de réinterroger cette mise en place de l'identité du sujet, et dans certains cas de la faire vaciller. Ceci aura pour autre conséquence de faire osciller ces sentiments d'appartenance et de filiation qui sont primordiaux pour l'enfant. On est toujours l'enfant de quelqu'un, car comme le rappelle B. Cyrulnik: "N'appartenir à personne, c'est devenir personne". ${ }^{16}$

Les questions qu'inconsciemment l'enfant va se poser, comme tentative de réponse à ces violences inconscientes sont les suivantes:

- "A quel désir mon père, ma mère ont-ils obéi et quelle jouissance ont-ils privilégiée quand ils se sont séparés?"

- "Quelle jouissance a présidé à leur nouvel union?"

Dans la bouche de l'enfant, ceci peut devenir: “qu'est-ce que ce beau-parent a de plus et de mieux que le parent quitté? Est-ce à lui que je dois ressembler pour appartenir à ma deuxième famille? Je suis l'enfant de qui?"

\footnotetext{
${ }^{14}$ Quignard P., Le sexe et l'effroi. Paris, Gallimard, 1994, pp. 7-8.

${ }^{15}$ Winter J.P. L'érotique dans les famille recomposées" In Steichen R. et De Neuter P., op. cit., pp. 81-93.

${ }^{16}$ Cyrulnik B., Les nourritures affectives, 1993, O. Jacob, p 82.
} 
Questions inévitables, puisque c'est la mort du premier couple et donc de la première famille qui est le lit du second couple et de la seconde famille. Dans la mesure ou la séparation familiale répond à un voeu de mort de l'enfant sur l'un de ses parents ou sur les deux, (voeu de mort dans certain cas, inhérent à la problématique oedipienne), l'enfant va réagir soit sur un mode dépressif dû à sa culpabilité, soit sur un mode conflictuel et haineux qui fera surgir la violence.

\section{Pourquoi?}

Parce que le beau-parent, sans le savoir, vient dire à l'enfant que son voeu mortifère est réalisé. Confronté à cette réalisation, l'enfant ne peut que se défendre pour garder son appartenance nécessaire à sa vie et cette relation d'amour et de sécurité dont il a besoin.

Par ailleurs, si conjointement à la remise en question de son appartenance familiale, il lui est également demandé de choisir le parent qui aura sa garde, l'enfant est placé dans une position impossible. D'une part, les fonctions parentales lui sont abusivement attribuées et d'autre part à la culpabilité résultante de ses voeux mortifères va s'adjoindre celle que produit la problématique oedipienne. Voilà, cet enfant pris dans un noeud gordien indénouable.

C'est très probablement à ces endroits que se séparent irréductiblement le point de vue de la psychanalyse et le point de vue juridique. A mon avis, c'est aux parents et en cas d'impossibilité au juge à prendre cette décision. Il n'y a pas lieu de se décharger de cette décision sur l'enfant.

Le beau-parent qu'il le veuille ou non, du fait de la place qu'il occupe dans cette reconstruction familiale, est pour l'enfant, le support voire la cause de ces violences. Celles qui lui sont faites et celles que lui-même, enfant, va infliger aux adultes comme mécanismes de défense. Citons, par exemple: le rejet du beau-parent, le refus de se faire autre à l'image du nouveau couple, ou encore la reconstruction de son existence à partir de ce "traumatisme", " je suis un enfant de divorcé donc...". Christian Dubois appelle cette tentative de nomination "nomination réelle" en ce sens qu'elle s'appuie sur un événement réel. Ce type de nomination exigent pour Ch. Dubois, deux remarques. "D’une part, ce moment de déliaison du couple parental où chacun des parents était le garant de l'identification de l'enfant - déliaison au sens freudien de la pulsion de mort - va demander à être subjectivisé, c'est à dire intégré par l'enfant dans son "mythe individuel", et être aussi historisé. D'autre part, ce moment, que je qualifierais de crise subjective, va nécessiter de la part de l'enfant un travail pour retrouver ses points de repères identificatoires. Il s'agit d'une réitération des étayages narcissiques par un questionnement adressé aux parents ainsi qu’à ceux qui en prennent la place."

${ }^{17}$ Dubois Ch., L'autre démultiplier ou la quadrature du cercle. In Steichen R. et De Neuter P., op. cit., p. 240. 
La relation enfant beau-parent s'établira sur ces fondements et en véhiculera les enjeux. Ces enjeux sont très souvent insus aux acteurs. Ils n'ont pas ou peu de prise sur eux.

- Violence non sue, non voulue et cependant très réelle.-

Ces enjeux qui prendront une place d'autant plus grande que le beau-parent éprouve des difficultés à se situer dans cette relation, "puisqu'il n'est ni un parent, ni un parent de substitution", comme le souligne très justement M. Th. Meulders et I. Théry. ${ }^{18}$

Il vous est aisé de remarquer ici, combien la tâche du beau-parent est délicate et extrêmement difficile, s’il veut déjouer tous ces pièges:

- pièges du fait de la place qu'il occupe

- pièges du fait de la subjectivité de l'enfant et de son histoire

- pièges du fait de sa propre subjectivité et des désirs, raisons qui l'ont déterminé dans ce choix conjugal et familial.

Vous comprendrez aussi combien les premières relations qui vont s'établir entreeux pourront soit déjouer ces intrications des violences, soit les enflammer. Comment les déjouer ou, plus exactement, comment ne pas leur donner une consistance supplémentaire? Je pense que cela peut se faire par la reconnaissance par le beau-parent de ce qui a eu lieu et de ce que l'enfant vit toujours comme une très grande souffrance voire pour certain comme un traumatisme. Reconnaître que cette souffrance est une part de l'enfant, qu'elle est digne d'estime et capable d'apporter des éléments précieux pour construire l'avenir. Freud parlait de "réconciliation" et de "tolérance" du sujet à l'égard de sa propre souffrance. J'ajouterai: à l'égard de sa propre violence.

Cela peut aussi passer par la reconnaissance et l'acceptation de ce que, comme adulte, il ne peut rien comprendre de cette souffrance, ni la partager puisqu'il est étranger à cette famille désunie, mais aussi parce qu'il est souvent ou toujours pour l'enfant imaginairement la cause de cette désunion.

Peut encore avoir un effet positif, l'acceptation, en tant qu'il est légitime, voire nécessaire, du refus de l'enfant à son égard. Cette position du beau-parent apaisera l'enfant dans sa crainte d'être rapté dans ses origines, dans son intégrité psychique, son identité et dans la place qu'il occupe dans l'économie psychique de ses parents.

Cela implique que le beau-parent ne demande pas à l'enfant d'être aimé et reconnu par lui. Car cette demande peut subrepticement inclure la demande que l'enfant se déprenne à des degrés divers, de ses origines; voeux qu'exprimait un beau-père en ces termes: "Je sais que ne suis pas son père. Et ma femme tenait beaucoup à ce que je respecte

${ }^{18}$ Meulders M.Th. et Théry I., Les recompositions familiales aujourd'hui. Essais et recherches. Paris, Nathan, 1993. 
la relation à son père. Elle a tout fait pour cela. Mais au fond de moi, je n'ai qu' un voeu celui de l'éliminer et d'adopter cet enfant".

Cela implique aussi que le beau-parent n'exerce pas un rapport de séduction érotique sur l'enfant par un trop de gentillesse ou de laisser faire. Car quelle sera la limite entre la séduction de la violence et la violence de la séduction! Il ne conviendrait pas que par l'érotisation de sa relation à l'enfant et de sa demande d'être aimé de lui, il lui signifie qu'il n'est pas son père ou sa mère, ce qui est vrai. Il oublierait ainsi qu'il a à soutenir - partiellement - la fonction paternelle ou maternelle et que l'enfant ou l'adolescent confronté à la désintégration des imagos parentales va mettre en demeure ce nouveau conjoint d'un de ses parents de répondre de la validité de l'interdit de l'inceste parce qu'ils occupent une place parentale bien que ces enfants ne soient pas du même sang. ${ }^{19}$

Cette attitude d'apaisement que le beau-parent peut exercer à l'égard de l'enfant dans la mesure où il ne demande pas reconnaissance de sa place et fonction permettra entre autre à l'enfant de faire l'économie d'une mise en place de mécanismes de défenses pour maintenir son intégrité psychique et parfois physique. Mécanismes qui s'expriment en ces termes:

1. Ce qui est arrivé n'a pas eu lieu: processus “d'annulation” dirait Freud.

2. Il s'est bien passé quelque chose, mais cela n'a pas d'importance: processus d'isolation" dirait Freud.

Si l'enfant est ainsi refusé comme représentant et héritier de l'histoire de ses parents, il ne peut réagir à cette annulation ou à cette isolation que par un surgissement de violence.

\section{CONCLUSIONS}

Pour conclure, rappelons que la violence règne là où la parole se démet et là où elle est rendue impossible. Rappelons aussi que l'expérience clinique nous indique qu'il ne suffit pas toujours de parler. Que les enfants ou les adultes parlent de ce qu'ils ont vécu ne suffit pas toujours à faire disparaître les effets traumatiques des violences subies. Comme nous avons pu le constater, lorsque ces effets sont inscrits dans l'inconscient, ils ne s'amenuisent pas avec le temps, ils échappent aux possibles sublimations. La répétition ne les épuise pas, et leur oubli reste sans prise sur leur pouvoir pathogène. Enfin, la simple remémoration ne suffit pas à les faire disparaître. Agent ou victime de ces violences, les sujets le resteront à moins qu'ils puissent avoir accès à un lieu où ils pourront adresser leur parole à l'Autre, dans un rapport de dissymétrie subjective. En ce lieu, ils auront un possible accès à une autre prise sur le monde et sur eux-mêmes

${ }^{19}$ Dubois Ch., in Steichen R. et De Neuter P., Les familles recomposées et leur enfants, op. cit., p. 244. 
que celles dont ils disposaient notamment parce qu'on la leur avait transmise. Dans ce lieu Autre, dans ce lieu nouveau, ils pourront vivre qu'ils ne sont pas seulement agent ou pas seulement une victime de ces violences mais qu'une place autre leur est possible: celle d'acteur de leur vie capable de conjoindre la nouvelle inscription familiale avec l'ancienne sans que celle-ci ne doive plus faire l'objet d'une annulation.

\section{REFERÊNCIAS}

CYRULNIK, B. Les nourritures affectives, 1993, O. Jacob.

DECUYPERE, A., Les nouvelles compositions familiales et la question du temps". In: STEICHEN, R. et De Neuter P., op. cit., p. 194.

DESCAMPS, M. La fonction parentale au risque des parentés plurielles. In: STEICHEN R. et De Neuter, P. (édit), Les familles recomposées et leurs enfants. Louvain-la-Neuve, Académia, 1995, pp. 145-146.

DUBOIS, Ch. in STEICHEN, R. et De Neuter P., Les familles recomposées et leur enfants, op. cit., p. 244.

DUBOIS, Ch. L'autre démultiplier ou la quadrature du cercle. In: STEICHEN, R. et De Neuter P., op. cit., p. 240.

FREUD, S. Sur la psychologie du lycéen. In Résultats, idées, problèmes. Paris, Puf, tome I, p. 230.

LACAN, J. Séminaire I. Leçon du 30 juin 1954. Seuil, 1975, p. 298.

LACAN, J. L'agressivité en psychanalyse, in Ecrits, Paris, Seuil, 1966, p.ll7.

LECLAIRE, S. On tue un enfant. Paris, Seuil, 1975, p. 11.

MEULDERS, M.Th. et Théry I., Les recompositions familiales aujourd'hui. Essais et recherches. Paris, Nathan, 1993.

QUIGNARD, P. Le sexe et l'effroi. Paris, Gallimard, 1994, pp. 7-8.

STRYCKMAN, N. Parents en crise, adolescent en "passe". In: La psychanalyse de l'enfant. Paris, Ed. Association freudienne, 1989, Tome 2, p. 56.

STRYCKMAN, N. Dis moi, miroir, suis-je la plus belle du Royaume? Communication à la Journée de fin d'année de l'Association freudienne de Belgique, à Bruxelles en juin 1993.

WINTER, J.P. L'érotique dans les famille recomposées" In: STEICHEN R. et De Neuter P., op. cit., pp. 81-93.

Recebido em agosto de 2009 Aprovado em setembro 2009 